\title{
Análise comparativa e avaliação de prioridades de competição em três cadeias de suprimentos do setor petroquímico
}

\author{
Brian Silva ${ }^{\mathrm{a}}$, Cristiano Finardi ${ }^{\mathrm{b}}$, Marcelo Forneck ${ }^{\mathrm{c}}$, Miguel Afonso Sellitto ${ }^{\mathrm{d} *}$ \\ abrian.silva@grupopredial.com.br, Unisinos, Brasil \\ bcrisfin@terra.com.br, Unisinos, Brasil \\ cforneck.marcelo@gmail.com, Unisinos, Brasil \\ d*sellitto@unisinos.br, Unisinos, Brasil
}

\begin{abstract}
Resumo
Este artigo analisa comparativamente e avalia qualitativamente prioridades de competição em três cadeias de suprimentos da indústria petroquímica, A, B e C. A pesquisa foi feita no segundo semestre de 2006. 0 artigo revisa definições sobre cadeias de suprimentos, destacando principalmente a forma de rede que estas podem assumir. A seguir, apresenta os três casos estudados. A pesquisa identificou sete prioridades de competição em petroquímica: qualidade, entrega, flexibilidade, custo, inovação, comunicação e segurança, que foram desdobradas em dimensões. As prioridades foram ponderadas por gestores, com apoio do método multicriterial AHP, chegando-se a uma estrutura de competitividade, que apoiou a avaliação, baseada em julgamento. Ao fim, as diferenças entre as prioridades e os desempenhos julgados formaram uma estrutura de objetivos para as cadeias. A deve priorizar qualidade, B flexibilidade e $\mathrm{C}$ redução de custo.
\end{abstract}

Palavras-chave

Cadeia de suprimentos. Estratégias de produção. Critérios de competição. Competição em petroquímica. Medição de desempenho em SC.

\section{Introdução}

A competição nos mercados globais, menores ciclos de vida de produtos e expectativas aumentadas de clientes têm estimulado empresas de manufatura a procurar novas formas de arranjos produtivos. Uma destas formas é a cadeia de suprimentos (supply chain, SC). Para o SCC (SUPPLY..., 2007), uma SC reúne todos os esforços envolvidos na produção e entrega de produtos a clientes finais, compreendendo desde 0 primeiro fornecedor de fornecedor até o último cliente de cliente. 0 conselho estrutura estes esforços em: planejamento da cadeia, abastecimento de materiais e serviços, fabricação e montagem de bens, entrega, e retorno de resíduos e retalhos. Apenas o cliente final aporta novos recursos financeiros ao arranjo: nas demais operações, o dinheiro apenas troca de mãos entre participantes.
Lamming et al. (2000) argumentam que a palavra cadeia não reflete exatamente o que ocorre no arranjo. Os autores consideram que o sentido seria mais bem apreendido por uma eventual expressão rede de suprimentos (supply network). Harland, Lamming e Cousins (1999) diferenciam graficamente operações em cadeia de operações em rede, incluindo não linearidades, tais como ultrapassagens (por exemplo, o terceiro estágio abastecido diretamente pelo primeiro), recursividades (o segundo estágio alimenta o terceiro que retorna para o segundo) ou fluxos reversos. Christopher (2007) assume posição em favor de rede, argumentando que há, ao redor da empresa focal, múltiplos fornecedores, fornecedores de fornecedores, múltiplos clientes e clientes de clientes, em ligações reticulares, não lineares. Um 
grafo que as representasse se assemelharia mais a uma rede do que a uma cadeia. Kwok e Wu (2009) usam supply chain network para definir um conjunto de organizações conectadas que operam juntas para responder rápida e corretamente a demandas de clientes. Lamming et al. (2000) admitem que o termo cadeia de suprimentos tornou-se tão difundido que é difícil propor seu abandono. Pires (2004) pondera que se pode usar alternativamente tanto cadeia como rede de suprimentos.

Acrescenta-se ao debate que o termo cadeia pode ser mantido, desde que entendido como uma sequência linear de funções (fornecimento, fabricação, montagem, distribuição, entrega, consumo), não de empresas ou operações. Neste caso, o formato de cadeia prevalece se a sequência das operações é mantida. Pode-se inclusive argumentar que, se a sequência não for mantida, havendo, por exemplo, retornos ou ciclos (fabricação - montagem - fabricação), trata-se de outro tipo de arranjo que requererá outras técnicas de análise. Tal situação foi observada em um caso na indústria calçadista. A empresa focal recebe matériaprima petroquímica, processa-a, gera resina, devolve ao fornecedor para que gere pigmento e recebe de volta para a montagem do calçado.

Neste artigo, usou-se o termo cadeias de suprimentos (SC) para descrever o esforço conjunto de diversas empresas, cujo objetivo compartilhado é atender clientes finais, atingindo objetivos parciais, regulados por relações internas entre os participantes. SCs passaram a fazer parte da estratégia de produção de empresas focais, aquelas empresas que detêm, assimetricamente, mais capacidade de investimento e exercem mais governança no arranjo que as demais. Parcerias e alianças sinérgicas têm sido observadas em SCs. Empresas de manufatura, por exemplo, têm direcionado atividades de fabricação e submontagens a arranjos de fornecedores, cabendo às empresas apenas a gestão dos resultados e as competências principais do negócio (ANDRADE; FURTAD0, 2005). A função compras passou a subordinar-se diretamente à estratégia de fabricação (KRAUSE; SCANELL; CALANTONE, 2000; KRAUSE; PAGELL; CURKOVIC, 2001).

0 objetivo deste artigo foi analisar comparativamente e avaliar as prioridades de competição de três cadeias de suprimentos. 0 método de pesquisa foi o estudo de três casos em manufatura na indústria petroquímica, realizado no segundo semestre de 2006. Análise e comparação ocorreram com base na avaliação de prioridades escolhidas por especialistas em gestão de cadeias de suprimentos petroquímicas, em sessão de grupo focado. Em seguida, os gestores das cadeias ponderaram as prioridades por um método de apoio à decisão, o AHP, e avaliaram qualitativamente seu desempenho. Ao fim, chegou-se a um indicador final de desempenho qualitativo para cada cadeia, que permitiu comparações e gerou as principais lacunas de desempenho, que passaram a ser os principais objetivos de cadeia. A principal contribuição do artigo para o estudo de cadeias de suprimentos é o aprofundamento, por meio de mais casos, de um método já apresentado em Sellitto e Mendes (2006) de avaliação qualitativa de desempenho estratégico em SC. Escolheu-se o método pelo baixo custo de execução e por considerar opiniões e percepções de gestores estrategistas, que podem não ser percebidas de modo integrado em medições objetivas por grandezas de campo.

Medições e avaliações de desempenho em SC têm sido tema de pesquisas. Beamon (1999) apresentou e classificou indicadores usados em SC. Lambert e Pohlen (2001), Gunasekaran, Patel e Tirtiroglu (2001), Gunasekaran, Patel e McGaughey (2004) e Sellitto, Borchardt e Pereira (2011) desenvolveram quadros de trabalho, Lai, Ngai e Cheng (2002) construíram um sistema com vinte e seis indicadores, Kleijnen e Smits (2003) usaram o BSC, Chan e Qi (2003) usaram um método baseado nos processos da SC e Chan (2003) usou o AHP de modo próximo ao ora utilizado para priorizar dimensões de desempenho em SC, extraídas de referenciais em estratégia de operações: custo; eficiência; qualidade; flexibilidade; velocidade na inovação; confiabilidade e intensidade de inovação.

0 restante do artigo traz: revisão sobre gestão da cadeia de suprimentos e prioridades de competição em SC, metodologia de pesquisa, apresentação dos resultados de pesquisa, discussão dos resultados e considerações finais.

\section{Gestão da cadeia de suprimentos}

SCs englobam atividades associadas à movimentação e transformação de matérias-primas em bens acabados, desde as fontes primárias até o consumidor final, passando por operações de fabricação, montagem, armazenagem e distribuição (THOMAS; GRIFFIN, 1996). O SSC (SUPPLY..., 2007) acrescentou a função retorno de materiais. A SC envolve mais do que processos logísticos: envolve todos os processos de negócio requeridos para atender com competitividade o cliente final (SIMCH-LEVI; KAMINSKI; SIMCH-LEVl, 2003). Para Pires (2004), a SC é um conjunto de empresas autônomas, ou semiautônomas, que assume a responsabilidade pela obtenção, produção e liberação de produtos e serviços ao cliente final.

Bowersox, Cooper e Closs (2006) ilustram a SC por um diagrama sequencial que inter-relaciona as 
empresas participantes de uma unidade competitiva coordenada, formando um arranjo interorganizacional em que fluem materiais e informações. Os autores afirmam que a integração de operações na SC fica entre a integração vertical completa e a autonomia individual plena, pois partes individuais têm autonomia para perseguir objetivos globais. A Figura 1 apresenta uma SC composta pela empresa focal, fornecedores de primeira e de segunda camada a montante e distribuidores, varejistas e clientes finais a jusante.

Autores conceituaram e contextualizaram o gerenciamento da cadeia de suprimentos (Supply Chain Management - SCM). Para Ballou (2006), o SCM é a integração das atividades relacionadas com fluxo e transformação de materiais e informações na SC. Para Taylor (2006), o SCM deve ser distribuído na SC e não localizado nas empresas individuais, balanceando satisfação dos clientes e eficiência. Para Pires (2004), o SCM deve objetivar a conquista de uma vantagem competitiva sustentável pela coordenação estratégica e tática das funções de negócios, gerenciando o desempenho das empresas isoladamente e da cadeia como um todo. O SCM é multifuncional e abrange múltiplos interesses, integrando processos de negócios desde o usuário final até os fornecedores primários. 0 autor sugere diretrizes gerenciais: (i) compartilhamento de informações ao longo da $\mathrm{SC}$, especialmente às referentes ao planejamento e monitoramento de processos; (ii) divisão de riscos e ganhos, viabilizando relações de longo prazo; (iii) colaboração entre as empresas e compartilhamento de objetivos; (iv) integração nos processos de abastecimento, manufatura e distribuição; e (v) parcerias de longo prazo. Quanto ao último item, Guimarães, Cook e Natarajan (2002) apontam que é possível reduzir custos mantendo poucos parceiros fortemente integrados e com altas escalas de produção.

Para Simchi-Levi, Kaminski e Simch-Levi (2003), o SCM é um conjunto de abordagens utilizadas para integrar eficientemente fornecedores, fabricantes e distribuidores, de forma que a mercadoria seja produzida e distribuída na quantidade certa, para a localização certa e no tempo certo, minimizando os custos globais do sistema e atingindo o nível de serviço desejado. Para Christopher (2007), o SCM é a extensão do gerenciamento logístico interno para a cadeia externa de fornecimento. Esforços de redução de tempos de atravessamento, de níveis de estoques e de aumento de qualidade produzem ganhos de competitividade compartilhados pelos participantes. 0 SCM inclui práticas e iniciativas logísticas que integram empresas e favorecem o fluxo de informações. 0 autor resume (CHRISTOPHER, 2007, p. 4):

“[...] a gestão das relações a montante e a jusante com fornecedores e clientes, para entregar mais valor ao cliente, a um custo menor para a cadeia de suprimentos como um todo.”.

\section{Prioridades de competição em SC}

A noção de prioridades de competição tem sido usada como instrumento de operacionalização de estratégias e tem sido um recurso para pesquisa e análise de estratégias de operações (WARD et al., 1998). Mesmo havendo diversas funções na SC, estratégias unificadas são necessárias para promover a integração das estratégias funcionais e perseguir prioridades compartilhadas (KRAUSE; PAGELL; CURKOVIC, 2001). Uma estratégia de SC é composta por decisões que cruzam as fronteiras das empresas e tem por objetivo atender demandas de clientes (HARLAND; LAMMING; COUSINS, 1999). Estratégias operacionais em SC são decisões que norteiam as operações de aquisição, abastecimento, fabricação, montagem, distribuição e retorno de produtos e serviços, que devem estar alinhadas à estratégia global da cadeia.

Watts, Kim e Hahn (1992) reconhecem que, para aperfeiçoar o desempenho da SC, estratégias funcionais de compras, de produção e de distribuição devem ser formuladas em conjunto. Como as operações são realizadas por empresas diferentes, não basta compartilhar o processo de formulação estratégica: é necessário alinhar o conteúdo. A execução de uma estratégia funcional de um membro da SC não deve prejudicar, e se possível, deve facilitar a execução de estratégias dos demais membros. É o gerenciamento das relações entre as estratégias que constrói e sustenta a vantagem competitiva da SC, principalmente se esta for capaz de promover

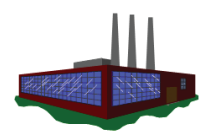

Fornecedor de segunda camada

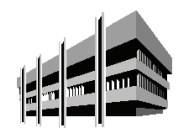

Fornecedor de primeira camada

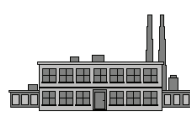

Empresa focal

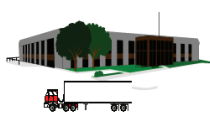

Distribuidor

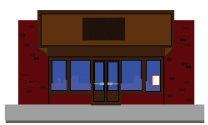

Varejista

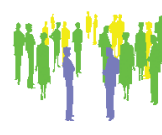

Cliente final 
a cooperação interorganizacional em prioridades dinâmicas (CHEN; PAULRAJ; LADO, 2004; CHEN; PAULRAJ, 2004; LORENZONI; LIPPARINI, 1999)

Pires (2004) teme que não seja possível formular e analisar uma estratégia unificada em SC, pois pode haver predominância de estratégias individuais. Para o autor, a estratégia da empresa focal pode influenciar as estratégias de membros do arranjo. Adicionalmente, a participação das empresas em várias SC dificulta a implementação de uma estratégia unificada. Ainda assim, Krause, Pagell e Curkovic (2001) descrevem relações entre estratégias de operações de aquisição, fabricação e distribuição em SC. Os autores sugeriram cinco prioridades de competição que dependem, assimetricamente, de ações locais na SC: custo; qualidade; entrega; flexibilidade e inovação. Exemplificando a assimetria, um objetivo de qualidade depende mais das estratégias de aquisição ou de fabricação do que da de distribuição. 0 oposto ocorre em um objetivo de entrega. No entanto, problemas de entrega podem nascer na aquisição ou na fabricação, por exemplo, se o tipo de produto ou embalagem for de difícil embarque nos veículos usuais da SC. Na mesma linha, Harland, Lamming e Cousins (1999) resenham pesquisas com empresas, tais como Toyota, Nike, Benneton, Nissan, McDonald e Apple, que apontam a existência de gerenciamento estratégico interorganizacional, com ações e estratégias que cruzam as fronteiras das empresas.

Uma parte do processo estratégico em SC é a medição ou avaliação do desempenho. Sellitto e Mendes (2006) diferenciam medição de avaliação. Medição se origina de variáveis físicas objetivas medidas diretamente em campo ou calculadas a partir das medidas. Avaliação se origina de classificação e julgamento de decisores sobre aspectos subjetivos. A avaliação pode ser numérica, se for possível classificá-la em categorias, tais como: péssimo, ruim, médio, bom ou ótimo, associáveis a números. Para os autores, a medição produz um resultado objetivo, porém considera menos aspectos na mensuração. Já uma avaliação pode variar segundo a perspectiva do julgador, mas o julgamento pode incluir mais aspectos na mensuração. Neste artigo, tratou-se exclusivamente de avaliação de desempenho.

Para Pires (2004), a avaliação do desempenho é um processo que quantifica uma ação. A avaliação é processo de quantificação e a ação é o que leva ao desempenho. Em SCs, o autor sugere que se use um modelo de medição que se alinhe e integre os objetivos estratégicos da cadeia. Para Beamon (1999), o modelo de medição em SC inclui: o que medir; qual a frequência de medição; como integrar as medições; como usar as medições para controlar o desempenho; e como reavaliar o sistema de medição.
Lambert e Pohlen (2001) afirmaram na época que ainda era necessária pesquisa até que surgissem abordagens úteis na medição de desempenho em SC. Os autores atribuíram esta carência, entre outras causas, à complexidade que surge quando múltiplas partes individuais formam um arranjo que busca objetivos compartilhados. Algumas pesquisas feitas desde então foram sintetizadas em Sellitto, Borchardt e Pereira (2011). Seus métodos foram classificados em três grupos: (i) estruturas extraídas da teoria dos sistemas para descrever os construtos medidos; (ii) combinação de indicadores de empresas individuais com indicadores de ação sistêmica da cadeia; e (iii) modelos prescritivos que adotam algum tipo das chamadas melhores práticas, tais como o modelo SCOR. Combinações das três abordagens primitivas também foram observadas. 0 modelo usado neste artigo está mais próximo do terceiro grupo.

\section{A pesquisa: método e resultados}

0 tema da pesquisa foi: avaliação de desempenho e análise de competitividade em SC. A questão de pesquisa foi: como avaliar qualitativamente, para comparação e análise estratégica, o desempenho de cadeias de suprimentos em prioridades de competição? 0 objetivo de pesquisa que responde à questão foi aplicar um método para avaliar qualitativamente e analisar prioridades de competição em SC. 0 método de pesquisa escolhido foi o estudo de três casos em três SCs de bens manufaturados do setor petroquímico. A escolha é coerente com Yin (2001): questões do tipo como remetem ao estudo de caso. Para Eisenhardt (1989), a repetição de casos pode encaminhar os achados de pesquisa em direção a uma teoria indutivamente fundamentada. Nesta pesquisa, os três casos referem-se a cadeias distintas, mas esperava-se que algumas regularidades pudessem surgir na análise delas. Os casos foram do tipo descritivo: mais do que uma exploração da realidade das SC, mas ainda sem chegar às causas dos fenômenos observados, limitando-se à sua descrição e análise. A contribuição essencial do artigo é a descrição de três situações heurísticas criadas para teste e reforço de uma ideia teórica e a emersão de regularidades que possam contribuir para uma teoria sobre o tema. Os casos seguem a linha de pesquisa iniciada em Sellitto e Mendes (2006) e possibilitam o surgimento de mais particularidades a contemplar na teoria.

0 método de trabalho foi: (i) identificação de três SCs da indústria petroquímica e visitas dos pesquisadores para exploração inicial; (ii) sessão de grupo focado com pesquisadores e seis especialistas em SC da indústria, na qual foi discutida competitividade no setor e da qual emergiram sete prioridades 
de competição, organizadas em vinte e cinco dimensões; (iii) alocação de um pesquisador a cada cadeia e condução de grupo focado com gestores estrategistas da cadeia para distribuir importância entre as prioridades de cada cadeia e avaliar, por escala categórica [péssimo $=0 ;$ ruim $=0,25 ;$ médio $=0,5$; bom $=0,75 ;$ ótimo $=1$ ], o desempenho das SC nas dimensões; e (iv) integrar os resultados, discutir comparativamente as avaliações e identificar as lacunas de desempenho, ou seja, que prioridades devem ser enfocadas, na avaliação dos respondentes, para aumento de competitividade na SC.

A distribuição de importância foi feita com o AHP (Analytic Hierarchy Process). As prioridades foram colocadas em ordem decrescente de importância e comparadas duas a duas. Para sete prioridades $a_{i}$, $i=[1,2, \ldots, 7]$, houve vinte e uma comparações em resposta às perguntas: Para $a_{i} a_{\vec{p}} \forall i, j$, dado que $a_{i}$ é mais importante do que $a_{j} a_{i}$ é: (i) um pouco mais importante? (ii) mais importante? (iii) muito mais importante? ou (iv) absolutamente mais importante do que $a_{j}$ ? As respostas foram transformadas em números segundo a Tabela 1. Valores intermediários atendem a situações intermediárias, nas quais foi necessário discernir entre duas alternativas próximas. Se $a_{i j}=p$, $a_{j i}=1 / p$; se $i=j, p=1$ (SAATY, 1991).

As respostas formaram a matriz de preferências $A$ (Equação 1). Pela Equação 2, calcula-se o autovetor de máximo autovalor da matriz, o vetor de prioridades w. 0 máximo autovalor $\lambda_{\max } \leq n(n=$ número de critérios) permite calcular a razão de consistência $C R$ (Equação 3), a medida de consistência do julgamento. Quanto mais próximos $\lambda_{\max }$ e $n$, mais consistente 0 resultado. $I R$ é o índice randômico médio, extraído de tabela obtida por simulações com amostras de matrizes $n$-dimensionais. A $C R$ representa a probabilidade que a matriz de preferências não tenha sido obtida por julgamento racional, mas por acaso. Para os proponentes do método, $C R<0,1$ indica julgamento satisfatório. A tabela de simulações e justificativas teóricas do método são encontradas em Saaty (1991).

$A=\left[\begin{array}{cccc}1 & a_{12} & \cdots & a_{1 n} \\ 1 / a_{12} & 1 & \cdots & a_{2 n} \\ \vdots & \vdots & : \vdots & \vdots \\ 1 / a_{1 n} & 1 / a_{2 n} & \cdots & 1\end{array}\right]$

$A . \mathrm{w}=\lambda_{\max } \times \mathrm{w}$

$C R=\left[\lambda_{\max }-n\right] /[I R \times(n-1)]$
Tabela 1. Opções de preferência em comparação pareada.

\begin{tabular}{|c|c|c|c|c|}
\hline $\begin{array}{c}\text { Se } a_{i} \mathrm{em} \\
\text { relação a } a_{j}=\end{array}$ & $\begin{array}{c}\text { Então } \\
c_{i j}=\end{array}$ & $\begin{array}{c}\text { Se } a_{i} \text { em relação } \\
\text { a } a_{j}=\end{array}$ & $\begin{array}{c}\text { Então } \\
c_{i j}=\end{array}$ & Descrição \\
\hline lgual & 1 & lgual & 1 & $\begin{array}{l}\text { Os dois critérios } \\
\text { equilibram-se }\end{array}$ \\
\hline $\begin{array}{l}\text { Um pouco mais } \\
\text { importante }\end{array}$ & 3 & $\begin{array}{l}\text { Um pouco menos } \\
\text { importante }\end{array}$ & $1 / 3$ & $\begin{array}{c}\text { Existe leve } \\
\text { favorecimento }\end{array}$ \\
\hline $\begin{array}{c}\text { Mais } \\
\text { importante }\end{array}$ & 5 & $\begin{array}{c}\text { Menos } \\
\text { importante }\end{array}$ & $1 / 5$ & $\begin{array}{l}\text { Existe forte } \\
\text { favorecimento }\end{array}$ \\
\hline $\begin{array}{l}\text { Muito mais } \\
\text { importante }\end{array}$ & 7 & $\begin{array}{l}\text { Muito menos } \\
\text { importante }\end{array}$ & $1 / 7$ & $\begin{array}{l}\text { Existe muito forte } \\
\text { favorecimento }\end{array}$ \\
\hline $\begin{array}{l}\text { Absolutamente } \\
\text { mais importante }\end{array}$ & 9 & $\begin{array}{l}\text { Absolutamente } \\
\text { menos importante }\end{array}$ & $1 / 9$ & $\begin{array}{l}\text { Existe dominância } \\
\text { absoluta }\end{array}$ \\
\hline
\end{tabular}

\subsection{Estrutura de competitividade}

Por conveniência de acesso, os pesquisadores escolheram três cadeias do setor petroquímico. Depois de contatos iniciais e visitas exploratórias, os pesquisadores conduziram uma sessão de grupo focado, da qual participaram quatro pesquisadores e seis especialistas praticantes gestores de cadeias do setor petroquímico, com formação superior (dois com mestrado) e experiência compatível com a pesquisa. A teoria de grupos focados e o método empregado são apresentados em Ribeiro e Neumann (2003). Quanto às SCs, comenta-se que o setor petroquímico é abastecido pelo mesmo grande fornecedor de matériaprima, a nafta, por sua vez abastecida por refinaria de petróleo. Uma representação possível é uma rede que nasce de caminho serial de duas grandes empresas, composta por diversas cadeias que se emaranham e se tocam em alguns pontos, lideradas por empresas focais com governança local. Esta imagem é coerente com a metáfora de Lamming et al. (2000): uma rede de suprimentos (supply network) composta por várias cadeias com algumas funções independentes, outras não. A Figura 2 traz um grafo que representa o cenário de pesquisa.

0 grafo simplifica a realidade. A área de interesse inicia na refinaria de petróleo que fornece nafta para a central de matérias-primas, que, por sua vez, fornece para as empresas de segunda geração, gerando resinas termoplásticas e outros produtos, sólidos e líquidos. Há uma estrutura de armazenagem, formada por operadores de serviços logísticos, que abastecem diversos tipos de processadores, que, por sua vez, abastecem diversas indústrias. Para o objetivo de pesquisa, interessaram a coureiro-calçadista, a da moda e a de utilidades. As três cadeias estudadas são caminhos dentro da rede com alguns pontos de contato.

0 grupo focado chegou à estrutura conceitual do Quadro 1, que pode representar prioridades de competição na indústria, composta por: qualidade, entrega, flexibilidade, custo, inovação e tecnologia, 


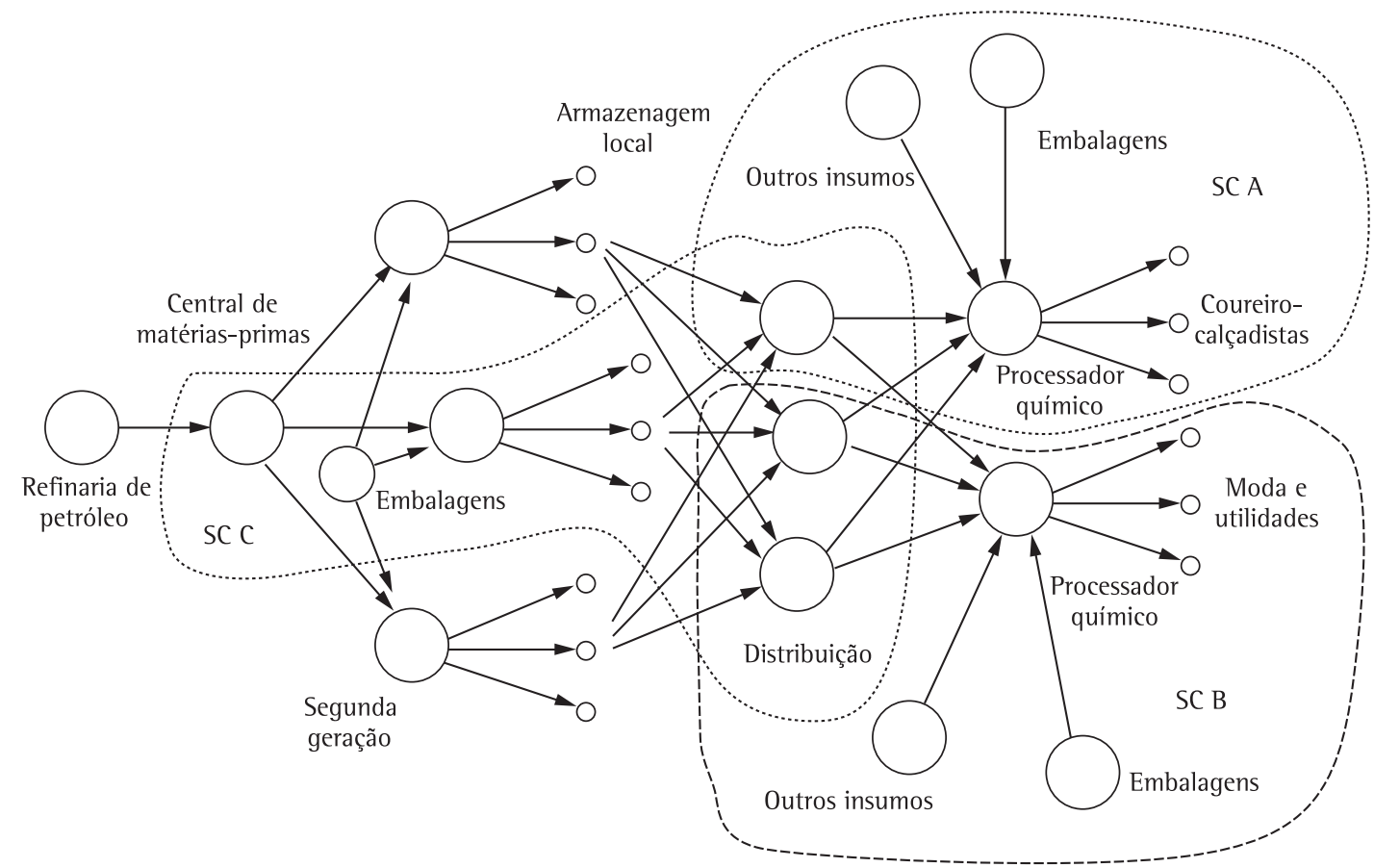

Figura 2. SCs estudadas e relações na indústria petroquímica.

comunicação e segurança. Krause, Pagell e Curcovic (2001) citam as quatro primeiras como recorrentes em estudos similares. 0 grupo observou que estas quatro decidem negócios: clientes compram ou não por sua presença ou ausência na SC. Inovação e tecnologia e comunicação foram descritas como prioridades intermediárias, que podem ser úteis para o atingimento das principais. Segurança é condição necessária: se não existe, pode prejudicar as prioridades principais.

As sete prioridades foram desdobradas em dimensões consideradas relevantes pelo grupo na descrição da competitividade das cadeias estudadas. Em qualidade, o grupo entendeu que deviam ser avaliadas: a confiabilidade do produto, o desempenho quanto à agregação de valor e à conformidade a especificações. Em entregas: a integralidade das cargas, o prazo prometido, a pontualidade e a variabilidade. Em flexibilidade: a capacidade de resposta rápida da SC para aumentar e diminuir os volumes e variar o mix de entregas. Em custos: o custo total, as informações sobre custos, a capacidade de negociação e o nível de preços praticados pela SC. Em inovação e tecnologia: a intensidade da inovação, a capacidade tecnológica aplicada nas operações, a gestão do conhecimento e o acesso ao conhecimento por parte dos membros da SC. Em comunicação: os recursos telemáticos da SC (EDI - Electronic Data Interchange), o acesso e troca de informações entre os membros (sistemas compartilhados, tal como VMl - Vendor Managed
Inventory) e a capacidade de incorporar a voz do cliente nas operações (parâmetros técnicos de projeto, tipo de embalagem, tipo de entrega, etc.). Quanto à segurança da operação, o setor trata com produtos de alto risco de dano ambiental. Acidentes relatados trouxeram consequências importantes ao ambiente e à imagem do setor. Foram avaliados a adequação da $\mathrm{SC}$ às normas e legislação, a ética ambiental, a especificação e uso de EPls e EPCs (equipamentos de proteção individual e coletiva), o treinamento e o estado dos equipamentos usados nas operações. Pressões de clientes finais têm forçado clientes intermediários a valorizar e exigir rígidas políticas de segurança pessoal, patrimonial e ambiental, sob pena de perdas de negócios.

\section{Resultados das SCs}

A SC A fornece insumos químicos de alto valor, alta diversidade e baixo volume à indústria coureirocalçadista. A cadeia recebe matéria-prima de fornecedor petroquímico, que recebe do grande fornecedor de nafta. A cadeia também recebe outros insumos e embalagens, inclusive importados, processando, embalando, armazenando e despachando para os clientes. Um pesquisador conduziu um grupo de cinco gestores da cadeia. Para aumentar a consistência, antes de montar a tabela, o grupo ordenou as prioridades. Deste modo, os valores das células acima da diagonal 
Quadro 1. Estrutura de competitividade para as SCs estudadas.

\begin{tabular}{|c|c|c|}
\hline Prioridade & Dimensão & Descrição \\
\hline \multirow{3}{*}{ Qualidade } & Qualidade intrínseca & $\begin{array}{c}\text { Como é a capacidade da SC em fornecer produtos e serviços de alta confiabilidade } \\
\text { e cujo uso agregue alto valor ao produto final. }\end{array}$ \\
\hline & Desempenho comparado & $\begin{array}{c}\text { Como é a capacidade da SC para projetar e oferecer produtos com } \\
\text { desempenho superior ao mercado. }\end{array}$ \\
\hline & Conformidade a especificações & $\begin{array}{l}\text { Como é a capacidade da SC em atender com produtos e serviços } \\
\text { conformes às especificações pré-definidas do cliente final. }\end{array}$ \\
\hline \multirow{4}{*}{ Entrega } & Integralidade das entregas & $\begin{array}{c}\text { Como é a probabilidade que o cliente final seja plenamente atendido } \\
\text { na integralidade e na especificação do pedido. }\end{array}$ \\
\hline & $\begin{array}{l}\text { Prazo de entrega típico } \\
\text { prometido }\end{array}$ & $\begin{array}{l}\text { Como é o tempo típico que a SC promete para } \\
\text { as entregas em relação ao mercado. }\end{array}$ \\
\hline & Pontualidade das entregas & Como é a confiabilidade em relação ao cumprimento das entregas nas datas prometidas. \\
\hline & Variabilidade das entregas & Como é a variabilidade do tempo de entregas da SC. \\
\hline \multirow{3}{*}{ Flexibilidade } & Aumento de lote & $\begin{array}{c}\text { Como é a capacidade, vontade e custos da SC para, em outras entregas, } \\
\text { aumentar um lote de produção consolidado. }\end{array}$ \\
\hline & Redução de lote & $\begin{array}{l}\text { Como é a capacidade, vontade e custos da SC para, em outras entregas, } \\
\text { reduzir um lote de produção consolidado. }\end{array}$ \\
\hline & Mix de entrega & $\begin{array}{l}\text { Como é a capacidade, vontade e custos da SC para atender } \\
\text { mudanças de mix de produtos já solicitados e ainda não entregues. }\end{array}$ \\
\hline \multirow{4}{*}{ Custo } & Custo total & Como é o total de custos da SC associados aos produtos perante o mercado. \\
\hline & Informações sobre os custos & Como é a capacidade da SC para detalhar, informar e gerenciar os custos dos produtos. \\
\hline & Negociação & $\begin{array}{l}\text { Como é o histórico da SC quanto à negociação de preços, } \\
\text { prazos e condições de pagamento com clientes. }\end{array}$ \\
\hline & Preços competitivos & Como são os preços historicamente praticados pela SC perante o mercado. \\
\hline \multirow{4}{*}{$\begin{array}{l}\text { Inovação e } \\
\text { tecnologia }\end{array}$} & Inovação de produtos & $\begin{array}{l}\text { Como é a capacidade da SC para desenvolver novos produtos ou } \\
\text { fazer alterações substanciais em produtos de linha. }\end{array}$ \\
\hline & Capacidade tecnológica & $\begin{array}{c}\text { Como é o nível da capacidade tecnológica empregada pela SC } \\
\text { em relação ao disponível no mercado. }\end{array}$ \\
\hline & Gestão do conhecimento & $\begin{array}{l}\text { Como é o modo com que a SC constrói conhecimento e } \\
\text { inteligência competitiva em relação ao negócio e ao mercado. }\end{array}$ \\
\hline & Acesso ao conhecimento & $\begin{array}{l}\text { Como é a habilidade, a vontade ou a intensidade } \\
\text { com que ocorre a partilha de conhecimento na SC. }\end{array}$ \\
\hline \multirow{3}{*}{ Comunicação } & Recursos utilizados & Como a comunicação interna ocorre na SC (meio físico, meio eletrônico, verbal). \\
\hline & Acesso e troca de informações & $\begin{array}{l}\text { Como é a habilidade, a vontade ou a intensidade com que } \\
\text { ocorre a troca de informações operacionais na SC. }\end{array}$ \\
\hline & Participação do cliente final & $\begin{array}{l}\text { Como é a rapidez e a sensibilidade da SC para adequação } \\
\text { de características do produto a requisitos de clientes. }\end{array}$ \\
\hline \multirow{4}{*}{ Segurança } & Normas e legislação & $\begin{array}{l}\text { Como é a adequação às normas de segurança e às } \\
\text { legislações municipais, estaduais e federais em vigor. }\end{array}$ \\
\hline & Ética Ambiental & $\begin{array}{l}\text { Como é o posicionamento da SC quanto à questão ambiental, } \\
\text { certificações e demais legislações vigentes. }\end{array}$ \\
\hline & EPls & $\begin{array}{l}\text { Como são detalhadas e qual o nível de informação e } \\
\text { controle na SC sobre uso de EPls e a presença de especialistas em segurança. }\end{array}$ \\
\hline & Orientação para operações & $\begin{array}{l}\text { Como são e qual o nível de detalhamento das informações de } \\
\text { segurança para manuseio, armazenagem e transporte dentro da SC. }\end{array}$ \\
\hline
\end{tabular}

resultam iguais ou crescentes. A Tabela 2 mostra os resultados. A $C R$ foi de 3,5\%, satisfatória. A Equação 4 sintetiza o o julgamento.

$$
\begin{aligned}
& A=0,396 \times[\text { Qualidade }]+0,194 \times[\text { Custo }]+ \\
& +0,172 \times[\text { Entrega }]+0,084 \times[\text { Inovação }]+ \\
& +0,08 \times[\text { Flexibilidade }]+0,05 \times[\text { Segurança }]+ \\
& +0,024 \times[\text { Comunicação }]
\end{aligned}
$$

A SC B fornece insumos químicos de baixo valor, média diversidade e médio volume à indústria de moda e tendências. A cadeia recebe matéria-prima do mesmo fornecedor petroquímico de A e outros insumos locais, processa e embala os materiais, armazenando e despachando. Um pesquisador conduziu o grupo de três gestores e chegou ao resultado da Tabela 3, com $C R=4,7 \%$, satisfatória. A Equação 5 sintetiza o julgamento.

$$
\begin{aligned}
& \mathrm{B}=0,397 \times[\text { Flexibilidade }]+0,206 \times[\text { Inovação }]+ \\
& +0,187 \times[\text { Entrega }]+0,095 \times[\text { Custo }]+ \\
& +0,055 \times[\text { Comunicação }]+0,036 \times[\text { Qualidade }]+ \\
& +0,023 \times[\text { Segurança }]
\end{aligned}
$$

A cadeia C tem como empresa focal um importante fornecedor de resinas termoplásticas para indústrias de base, tais como automotiva, calçadista, eletroeletrônica e plásticos. A empresa opera com médio valor, baixa diversidade e alto volume, recebe 
Tabela 2. Resultados da avaliação pareada na SC A.

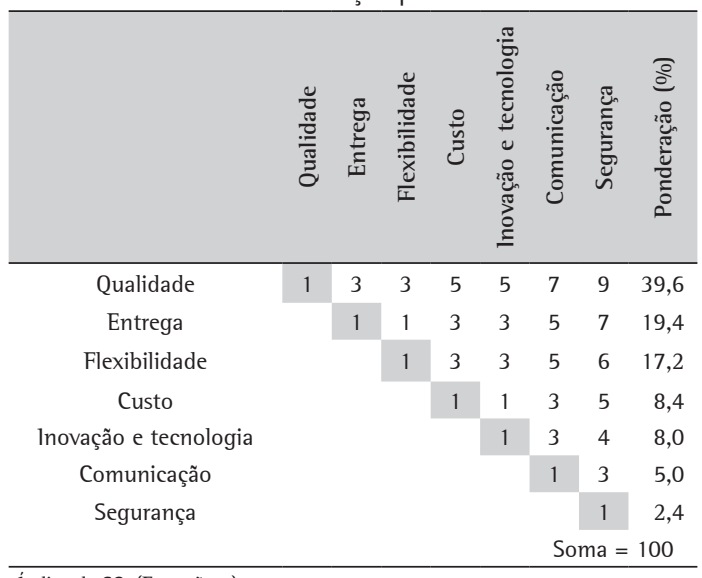

Índice da SC: (Equação 4).

Tabela 3. Resultados da avaliação pareada na SC B.

\begin{tabular}{|c|c|c|c|c|c|c|c|c|}
\hline & 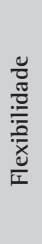 & 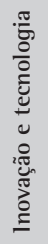 & 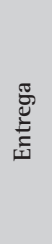 & $\stackrel{\frac{9}{n}}{3}$ & 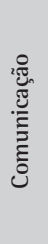 & $\begin{array}{l}\frac{\pi}{\pi} \\
\frac{\pi}{0} \\
\frac{0}{\pi} \\
\tilde{J}\end{array}$ & 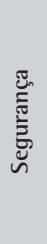 & 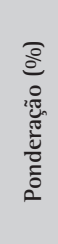 \\
\hline Flexibilidade & 1 & 3 & 3 & 5 & 7 & 8 & 9 & 39,7 \\
\hline Inovação e tecnologia & & 1 & 1 & 3 & 5 & 7 & 8 & 20,6 \\
\hline Entrega & & & 1 & 3 & 5 & 7 & 8 & 18,7 \\
\hline Custo & & & & 1 & 3 & 4 & 5 & 9,5 \\
\hline Comunicação & & & & & 1 & 3 & 4 & 5,5 \\
\hline Qualidade & & & & & & 1 & 2 & 3,6 \\
\hline \multirow[t]{2}{*}{ Segurança } & & & & & & & 1 & 2,3 \\
\hline & & & & & & \multicolumn{3}{|c|}{ Soma $=100$} \\
\hline
\end{tabular}

Índice da SC: (Equação 5).

do fornecedor de matéria-prima básica (nafta) e fornecedores de embalagens e serviços e entrega a uma rede de armazéns e distribuidores, conectados por transportadoras. 0 resultado do julgamento de cinco gestores é apresentado na Tabela 4. A razão de consistência foi $C R=5,5 \%$, satisfatória. A Equação 6 sintetiza o julgamento.

$$
\begin{aligned}
& C=0,361 \times[\text { Segurança }]+0,274 \times[\text { Qualidade }]+ \\
& +0,163 \times[\text { Custo }]+0,086 \times[\text { Inovação }]+ \\
& +0,054 \times[\text { Entrega }]+0,036 \times[\text { Flexibilidade }]+ \\
& +0,026 \times[\text { Comunicação }]
\end{aligned}
$$

\subsection{Desempenhos das SC}

Quanto ao desempenho atual, os pesquisadores estimularam os praticantes gestores das $\mathrm{SCs}$ a avaliarem a situação das dimensões, respondendo: Como está a situação da SC X em relação à dimensão $Y$ ? Depois de discussões, verificações de campo e consultas a indicadores próprios e externos, foi
Tabela 4. Resultados da avaliação pareada na SC C.

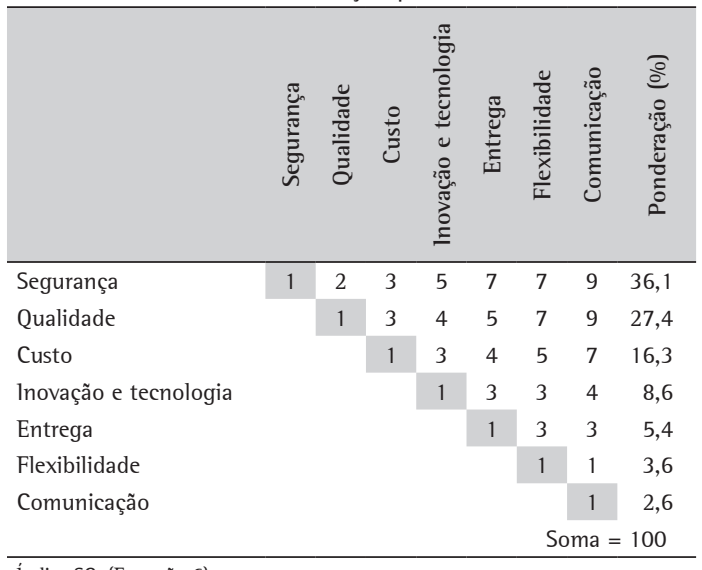

Índice SC: (Equação 6)

feito o julgamento, segundo a escala: [péssimo $=0$; ruim $=0,25 ;$ médio $=0,5 ;$ bom $=0,75 ;$ ótimo $=1]$. Multiplicando o valor do julgamento pela importância relativa, tem-se o desempenho da SC na dimensão. A soma dos desempenhos é o desempenho total da SC, que deve ficar entre 0 e $100 \%$.

0 resultado das avaliações surge na Tabela 5 . Nas duas primeiras colunas, tem-se a estrutura de competitividade. Para cada SC, seguem-se três colunas, contendo respectivamente a importância relativa das dimensões, obtidas dividindo-se linearmente a importância da prioridade pelo número de dimensões, o julgamento e o desempenho. Na última linha surgem os desempenhos relativos totais das três SCs.

\section{Discussão}

A observação da Tabela 5 permite considerações quanto às exigências de competitividade e os desempenhos percebidos pelos gestores. Inicia-se pela competitividade.

Apesar de as três SCs operarem na mesma indústria, operam negócios diferentes, com diferentes exigências de competição. 0 fator mais importante na SC A é a qualidade do produto entregue; na B é a flexibilidade; e na C é a segurança das operações. Não foi objetivo de pesquisa investigar as razões destas preferências, apenas descrevê-las, mas algumas considerações podem ser feitas.

A SC A fornece um insumo de alto valor, alta diversidade e baixo volume para a indústria coureirocalçadista. São produtos químicos para o recurtimento e tratamento de couros com alta participação na agregação de valor para o produto final, considerados estratégicos pelos compradores: sua presença ou ausência no produto final pode decidir negócios. As prioridades mais importantes são a qualidade 
Tabela 5. Análise comparada das três SCs.

\begin{tabular}{|c|c|c|c|c|c|c|c|c|c|c|}
\hline \multirow[b]{2}{*}{ Prioridade } & \multirow[b]{2}{*}{ Dimensão } & \multicolumn{3}{|c|}{$\mathrm{SC} A$} & \multicolumn{3}{|c|}{ SC B } & \multicolumn{3}{|c|}{ SC C } \\
\hline & & 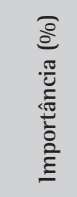 & 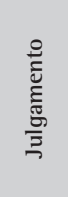 & 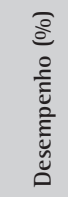 & 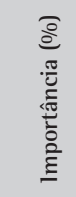 & 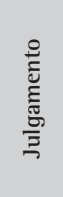 & 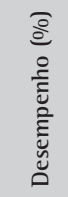 & 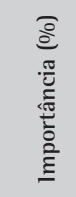 & 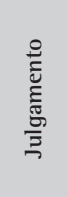 & 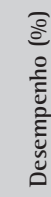 \\
\hline \multirow[t]{3}{*}{ Qualidade } & Qualidade intrínseca & 13,2 & 0,75 & 9,9 & 1,2 & 0,75 & 0,9 & 9,1 & 1,00 & 9,1 \\
\hline & Desempenho comparado & 13,2 & 0,75 & 9,9 & 1,2 & 0,50 & 0,6 & 9,1 & 0,75 & 6,9 \\
\hline & Conformidade a especificações & 13,2 & 0,75 & 9,9 & 1,2 & 0,50 & 0,6 & 9,1 & 1,00 & 9,1 \\
\hline \multirow[t]{4}{*}{ Entrega } & Integralidade das entregas & 4,8 & 0,75 & 3,6 & 4,7 & 1,00 & 4,7 & 1,3 & 0,50 & 0,7 \\
\hline & Prazo de entrega típico prometido & 4,8 & 0,75 & 3,6 & 4,7 & 0,75 & 3,5 & 1,3 & 0,50 & 0,7 \\
\hline & Pontualidade das entregas & 4,8 & 0,75 & 3,6 & 4,7 & 0,75 & 3,5 & 1,3 & 0,50 & 0,7 \\
\hline & Variabilidade das entregas & 4,8 & 0,50 & 2,4 & 4,7 & 0,75 & 3,5 & 1,3 & 0,25 & 0,3 \\
\hline \multirow[t]{3}{*}{ Flexibilidade } & Aumento de volume & 5,7 & 0,50 & 2,9 & 13,2 & 1,00 & 13,2 & 1,2 & 0,50 & 0,6 \\
\hline & Redução de volume & 5,7 & 0,50 & 2,9 & 13,2 & 1,00 & 6,6 & 1,2 & 0,25 & 0,3 \\
\hline & Mix de entrega & 5,7 & 0,50 & 2,9 & 13,2 & 0,50 & 6,6 & 1,2 & 0,50 & 0,6 \\
\hline \multirow[t]{4}{*}{ Custo } & Custo total & 2,1 & 0,25 & 0,5 & 2,4 & 1,00 & 2,4 & 4,1 & 0,75 & 3,0 \\
\hline & Informações sobre os custos & 2,1 & 0,50 & 1,0 & 2,4 & 0,75 & 1,8 & 4,1 & 0,75 & 3,0 \\
\hline & Negociação & 2,1 & 0,75 & 1,6 & 2,4 & 0,75 & 1,8 & 4,1 & 0,50 & 2,0 \\
\hline & Preços competitivos & 2,1 & 0,50 & 1,0 & 2,4 & 1,00 & 2,4 & 4,1 & 0,25 & 1,0 \\
\hline \multirow{4}{*}{$\begin{array}{l}\text { Inovação e } \\
\text { tecnologia }\end{array}$} & Inovação de produtos & 2,0 & 0,75 & 1,5 & 5,2 & 0,75 & 3,9 & 2,2 & 0,75 & 1,6 \\
\hline & Capacidade tecnológica & 2,0 & 0,75 & 1,5 & 5,2 & 0,50 & 2,6 & 2,2 & 1,00 & 2,2 \\
\hline & Gestão do conhecimento & 2,0 & 0,50 & 1,0 & 5,2 & 0,75 & 3,9 & 2,2 & 0,50 & 1,1 \\
\hline & Acesso ao conhecimento & 2,0 & 0,50 & 1,0 & 5,2 & 0,50 & 2,6 & 2,2 & 0,50 & 1,1 \\
\hline \multirow[t]{3}{*}{ Comunicação } & Recursos utilizados & 1,7 & 1,00 & 1,7 & 1,8 & 0,5 & 0,9 & 0,9 & 0,75 & 0,7 \\
\hline & Acesso e troca de informações & 1,7 & 1,00 & 1,7 & 1,8 & 0,75 & 1,4 & 0,9 & 0,50 & 0,4 \\
\hline & Participação do cliente final & 1,7 & 0,75 & 1,2 & 1,8 & 0,75 & 1,4 & 0,9 & 0,25 & 0,2 \\
\hline \multirow[t]{5}{*}{ Segurança } & Normas e legislação & 0,6 & 0,75 & 0,5 & 0,6 & 0,50 & 0,3 & 9,0 & 1,00 & 9,0 \\
\hline & Ética Ambiental & 0,6 & 0,50 & 0,3 & 0,6 & 0,50 & 0,3 & 9,0 & 1,00 & 9,0 \\
\hline & EPls & 0,6 & 0,75 & 0,5 & 0,6 & 0,50 & 0,3 & 9,0 & 1,00 & 9,0 \\
\hline & Orientação para uso & 0,6 & 0,50 & 0,3 & 0,6 & 0,50 & 0,3 & 9,0 & 1,00 & 9,0 \\
\hline & Soma $=$ & 100,0 & & 66,9 & 100,0 & & 76,5 & 100,0 & & 81,5 \\
\hline
\end{tabular}

do produto, principalmente a conformidade a especificações, pela exigência de certificado de análise, a regularidade das entregas e alguma flexibilidade, principalmente aumento e redução de volume. A cadeia opera com baixos estoques, devido aos preços elevados, sendo necessárias entregas constantes e confiáveis de pequenos lotes. 0 mix varia menos e seu controle é menos importante. 0 custo também tem importância e é influenciado por matérias-primas importadas sujeitas a variações cambiais. 0 setor tem tecnologias consolidadas e tradicionais, não podendo ser considerado intensivo em conhecimento ou sensível à inovação, portanto inovação e tecnologia contribuem menos para o resultado. Como a cadeia é compactada, com a maior parte das principais operações próximas geograficamente, e os volumes são pequenos e variam pouco, comunicação e segurança têm menos importância.

A SC B fornece um insumo de baixo valor, média diversidade e médio volume para a indústria da moda e utilidades, atendendo vários pequenos clientes.
São produtos básicos que entram na transformação e têm participação intermediária na agregação de valor para o produto final. 0 mercado final opera com itens de moda e as previsões de demanda são de baixa confiabilidade, pois o mercado é sensivel a variações e tendências, inclusive internacionais. A maior prioridade percebida foi a flexibilidade nas entregas. A SC precisa mudar com frequência e intensidade os tamanhos de lote. Como há variações nos produtos, acumular estoque seria de pouca valia, restando à SC agregar flexibilidade às operações por set-ups rápidos e postergação (postponement) da composição do produto final. É possível antecipar a produção de um conjunto básico de matérias-primas. A formulação final é feita no último momento possivel, em processo cujos set-ups são simples. Deste modo, é possível aumentar rapidamente o volume de entrega, pois as matérias-primas já estão prontas. Também é possivel reduzir o volume, pois as matérias-primas que não forem empregadas em um lote, o serão em outros. 0 mix de entregas também pode ser controlado 
pela estratégia de operação da SC. A SC enfatiza o desenvolvimento de novos produtos, pois o mercado preferencial é o da moda. A seguir, vem a entrega, correlacionada com a exigência de flexibilidade. Em seguida, vem o custo, não tão importante, pois, havendo componentes de moda na SC, é possível praticar preços mais elevados. A SC é compacta e a comunicação é considerada como processo de apoio. Os problemas observados têm sido de fácil solução. Como os problemas de conformidade e confiabilidade do produto estão bem resolvidos, a qualidade tem baixa prioridade. Por fim, a segurança também tem baixa prioridade, pois os volumes são pequenos, as distâncias são curtas e o produto é inerte, não havendo maior discussão sobre o tema.

A SC C trata de commodity de médio valor, baixa diversidade e alto volume, em mercado estável e contingenciável, ou seja, devido ao excessivo porte da operação e do investimento requerido, há poucas opções para os compradores. A SC pode controlar, dentro de limites, preços, custos, volumes e entregas. Deste modo, flexibilidade e entrega têm menor importância para a competitividade, pois os clientes têm menor poder de escolha. Como os fluxos são estáveis, a maior preocupação é a segurança da operação. Existe um histórico de acidentes em diversas operações pelo mundo que produziram um conjunto importante de normas e regulamentações que devem ser seguidas com rigor, sob pena de inviabilizar o negócio, tanto legal como operacionalmente. A segunda prioridade é a qualidade, principalmente quanto à conformidade a

Tabela 6. Distribuição dos pontos de importância entre as prioridades.

\begin{tabular}{cccccccc}
\hline & \multicolumn{7}{c}{ Posto } \\
\hline & $1^{\circ}$ & $2^{\circ}$ & $3^{\circ}$ & $4^{\circ}$ & $5^{\circ}$ & $6^{\circ}$ & $7^{\circ}$ \\
\hline Qualidade & 1 & 1 & & & & 1 & \\
Entrega & & 1 & 1 & & 1 & & \\
Flexibilidade & 1 & & 1 & & & 1 & \\
$\quad$ Custo & & & 1 & 2 & & & \\
$\quad$ Inovação & & 1 & & 1 & 1 & & \\
Comunicação & & & & & 1 & 1 & 1 \\
Segurança & 1 & & & & & & 2 \\
\hline
\end{tabular}

especificações. 0 produto deve operar misturado com produtos de outras SC, portanto a especificação é rigorosa. Quanto à prioridade custo, há menos de dez fornecedores de porte no Brasil, com pouco espaço para negociação de preços. Os custos são controlados pela tecnologia de fabricação e controle de perdas. Em inovação, os produtos são padronizados de alto volume, e a diferenciação se limita à modificação de características específicas para novas aplicações, tais como resistência física ou a temperatura e a flexibilidade, prototipados em planta-piloto. Como a entrega é feita para distribuidores, os volumes são altos e a programação é estável, sendo esta uma baixa prioridade. A empresa trabalha com estoques elevados, portanto a flexibilidade que o mercado exige é plenamente atendida. Por fim, a comunicação é pouco representativa, pois a SC é compacta: as principais operações ocorrem em um raio de menos de trinta quilômetros, em distrito industrial com infraestrutura consolidada e atualizada.

A Tabela 6 sintetiza as exigências de competitividade nas cadeias, segundo os gestores. Qualidade, entrega e flexibilidade são as prioridades estratégicas que mais comparecem nos postos mais importantes. Custos e inovação comparecem em postos intermediários, enquanto que comunicação e segurança são as menos importantes.

0 desempenho nas prioridades é discutido de modo agregado (Tabela 7).

A Tabela 7 apresenta prioridades, desempenhos observados e lacunas (prioridade menos desempenho) para as SCs. As maiores lacunas apontam as maiores oportunidades de aumento de competitividade, segundo os gestores.

$\mathrm{Na}$ SC A, as prioridades são qualidade, flexibilidade e entrega. Custo e inovação representam oportunidades menores e comunicação e segurança são negligenciáveis. Qualidade de conformidade tem sido um problema, principalmente devido a fornecedores, que têm tido dificuldade em atender aos requisitos de produto. Como estes variam com frequência, exige-se desenvolvimento permanente de novas soluções, afetando fornecedores. Flexibilidade e entrega também têm sido afetadas por

Tabela 7. Lacunas de desempenho das três SCs.

\begin{tabular}{|c|c|c|c|c|c|c|c|c|c|}
\hline & \multicolumn{3}{|c|}{ SC A } & \multicolumn{3}{|c|}{$\mathrm{SC} B$} & \multicolumn{3}{|c|}{ SC C } \\
\hline & $\begin{array}{c}\text { Prioridade } \\
\qquad(\%)\end{array}$ & $\begin{array}{c}\text { Desempenho } \\
(\%)\end{array}$ & $\begin{array}{l}\text { Lacuna } \\
\qquad \%)\end{array}$ & $\begin{array}{c}\text { Prioridade } \\
\qquad(\%)\end{array}$ & $\begin{array}{c}\text { Desempenho } \\
(\%)\end{array}$ & $\begin{array}{l}\text { Lacuna } \\
\qquad(\%)\end{array}$ & $\begin{array}{c}\text { Prioridade } \\
\qquad(\%)\end{array}$ & $\begin{array}{c}\text { Desempenho } \\
(\%)\end{array}$ & $\begin{array}{c}\text { Lacuna } \\
(\%)\end{array}$ \\
\hline Qualidade & 39,6 & 29,7 & 9,9 & 3,6 & 2,1 & 1,5 & 27,4 & 25,1 & 2,3 \\
\hline Entrega & 19,4 & 13,3 & 6,0 & 18,7 & 15,2 & 3,5 & 5,4 & 2,4 & 3,0 \\
\hline Flexibilidade & 17,2 & 8,6 & 8,6 & 39,7 & 33,1 & 6,6 & 3,6 & 1,5 & 2,1 \\
\hline Custo & 8,4 & 4,2 & 4,2 & 9,5 & 8,3 & 1,2 & 16,3 & 9,1 & 7,1 \\
\hline lnovação & 8,0 & 5,0 & 3,0 & 20,6 & 12,9 & 7,7 & 8,6 & 5,9 & 2,7 \\
\hline Comunicação & 5,0 & 4,5 & 0,4 & 5,5 & 3,7 & 1,8 & 2,6 & 1,3 & 1,3 \\
\hline Segurança & 2,4 & 1,5 & 0,9 & 2,3 & 1,1 & 1,1 & 36,1 & 36,1 & 0,0 \\
\hline
\end{tabular}


problemas na estrutura logística da empresa, pois esta opera principalmente em mercados remotos, no extremo oriente. Inovação tem sido contemplada pela empresa focal e não é mais uma prioridade, pois sempre operou com produtos diferenciados, que decidem negócios de seus clientes. Na opinião dos gestores, este aspecto está bem atendido.

Na SC B, as prioridades são inovação e flexibilidade. Entrega é intermediária e as demais são negligenciáveis. Como tem trabalhado com produto básico, a cadeia tem se preocupado pouco com inovação e flexibilidade. Para os gestores, este parece ser um erro, já que seu principal mercado é o de moda, que privilegia pequenos lotes e inovação. Segundo os gestores, há risco de perda de espaço na indústria, caso a empresa focal não se preocupe um pouco mais com inovação e flexibilidade. 0 aspecto entrega surgiu devido à percepção de que a cadeia apresenta problemas logísticos, tais como insuficência de transporte, que algumas vezes prejudicam as entregas, principalmente as urgentes e de pequeno volume, requeridas pelas empresas de moda.

Na SC C, a prioridade é redução de custo. Qualidade, entrega, flexibilidade, inovação e comunicação são intermediárias. Segurança, segundo os gestores, está tão bem resolvida na SC que não é mais oportunidade de ganho de competitividade. Há consciência de clientes e fornecedores de que procedimentos específicos devem ser seguidos sempre, sob pena de acidentes de monta. Mais investimentos em segurança não trarão acréscimo de competitividade. Investimentos em redução de custo, que não têm sido priorizados, são a melhor oportunidade no momento, o que é coerente com operação de grande volume e baixa diversidade, pois permite explorar mais eventuais ganhos de escala.

Em sintese, recomenda-se que a SC A invista em aumento de qualidade e de flexibilidade, a B em inovação e aumento de flexibilidade e a $\mathrm{C}$ em redução de custo.

\section{Considerações finais}

O objetivo deste artigo foi analisar comparativamente e avaliar as prioridades de competição de três cadeias de suprimentos. 0 método foi o estudo de três SCs do setor petroquímico. Uma limitação de pesquisa é inerente ao método do caso: trata-se de expressões individuais do fenômeno. Outra é a limitação a avaliações qualitativas, que, ao integrar múltiplos fatores no julgamento, também carregam alguma subjetividade.

A pesquisa evidenciou que, embora operando no mesmo setor, as SC operam negócios com prioridades diferentes. Uma política unificada para o setor não garantiria bons resultados. Algumas operações, tais como a distribuição, atendem a mais de uma SC, com prioridades estratégicas diferentes e às vezes contraditórias. Tais contradições podem ser indícios do que afirma Pires (2004): "é difícil identificar uma estratégia unificada de cadeia, pois empresas individuais podem participar de várias SCs, com objetivos diferentes". Resta então formular uma população de planos para atender à diversidade de objetivos. Por exemplo, um operador logístico pode ser contratado para ser eficiente e seguro em uma operação, por exemplo, transportando lotes grandes e padronizados, e para ser ágil e flexível em outra, transportando lotes pequenos e aleatórios. 0 operador deve então ser capaz de formular planos diferentes para ambas as situações, algumas vezes usando o mesmo equipamento, porém com procedimentos diversos em cada caso.

A pesquisa acrescentou mais três casos à linha iniciada em Sellitto e Mendes (2006). Na continuidade, deve-se contextualizar o método dentro dos demais já propostos. Também se pode distribuir importância dentro das prioridades, já que se tem assumido distribuição uniforme no nível dos indicadores. Uma linha que pode ser seguida é a medição de resultados, que trataria de identificar indicadores quantitativos que apreendam, com uma dada confiabilidade, os construtos de competitividade assinalados, e colecionar os valores, formando séries históricas. Estas séries podem apontar a trajetória irreversível da SC como arranjo complexo e sua análise de longo prazo pode ser útil no entendimento das relações internas e com o cenário. Outra linha que pode ser seguida é a extensão da abordagem a outras SCs ou redes de empresas, desta vez não em manufatura, mas em serviços, tais como comunicações, distribuição de energia, transportes ou saneamento.

\section{Reconhecimento}

Deseja-se reconhecer a importância dos comentários dos revisores anônimos para o resultado final da pesquisa. Parte da pesquisa foi financiada pelo CNPq.

\section{Referências}

ANDRADE, C.; FURTADO, J. Discutindo processos de outsourcing da manufatura: uma análise a partir de elementos das indústrias eletrônica, farmacêutica e automobilística. In: ENCONTRO NACIONAL DE ENGENHARIA DE PRODUÇÃO - ENEGEP, 25., 2005, Porto Alegre. Anais... Porto Alegre, 2005.

BALlOU, R. Gerenciamento da Cadeia de Suprimentos/Logística Empresarial. Porto Alegre: Bookman, 2006.

BEAMON, B. Measuring supply chain Performance. International Journal of Operations \& Production Management, v. 19, n. 3, p. 275-292, 1999. http://dx.doi.org/10.1108/01443579910249714

BOWERSOX, D.; ClOSS, D.; COOPER, M. Gestão Logística de Cadeias de Suprimentos. Porto Alegre: Bookman, 2006.

CHAN, F. Performance Measurement in a Supply Chain. The International Journal of Advanced Manufacturing Technology, v. 21, n. 7, p. 534-548, 2003.

CHAN, F.; Q1, H. Feasibility of performance measurement system for supply chain: a process-based approach and measures. Integrated Manufacturing Systems, v. 14, n. 3, p. 179-190, 2003. http:// dx.doi.org/10.1108/09576060310463145 
CHEN, 1.; PAULRAJ, A.; LADO, A. Strategic purchasing, supply management, and firm performance. Journal of Operations Management, v. 22, n. 5, p. 505-523, 2004. http://dx.doi. org/10.1016/j.jom.2004.06.002

CHEN, 1.; PAULRAJ, A. Understanding supply chain management: critical research and a theoretical framework. Journal of Production Research, v. 42, n. 1, p. 131-163, 2004. http://dx.doi.org/10.1080/0 0207540310001602865

CHRISTOPHER, M. Logística e gestão da cadeia de suprimentos. São Paulo: Thomson Learning, 2007.

EISENHARDT, K. Building theories from case study research. Academy of Management Review, v. 14, n. 4, p. 532-550, 1989.

GUIMARÃES, T.; COOK, D.; NATARAJAN, N. Exploring the importanceof business clockspeed as a moderator for determinants of supplier network performance. Decision Sciences, v. 33, n. 4, p. 629-644, 2002. http://dx.doi.org/10.1111/j.1540-5915.2002. tb01659.x

GUNASEKARAN, A.; PATEL, C.; TIRTIROGLU, E. Performance measures and metrics in a supply chain environment. International Journal of Operations \& Production Management, v. 21, n. 1-2, p. 71-87, 2001. http://dx.doi.org/10.1108/01443570110358468

GUNASEKARAN, A.; PATEL, C.; McGAUGHEY, R. A framework for supply chain performance measurement. International Journal of Production Economics, v. 87, n. 3, p. 333-347, 2004. http:// dx.doi.org/10.1016/j.ijpe.2003.08.003

HARLAND, C.; LAMMING, R.; COUSINS, P. Developing the concept of supply strategy. International Journal of Operations \& Production Management, v. 19, n. 7, p. 650-675, 1999. http:// dx.doi.org/10.1108/01443579910278910

KLEIJNEN J.; SMITS M. Performance metrics in supply chain management. Journal of the Operational Research Society, v. 54, n. 5, p. 507-514, 2003. http://dx.doi.org/10.1057/palgrave. jors. 2601539

KRAUSE, D.; PAGELL, M.; CURKOVIC, S. Toward a measure of competitive priorities for purchasing. Journal of Operations Management, v. 19, n. 4, p. 497-512. 2001. http://dx.doi. org/10.1016/S0272-6963(01)00047-X

KRAUSE, D.; SCANELL, T.; CALANTONE, R. A structural analysis of the effectiveness of buying firms' strategies to improve supplier performance. Decision Sciences, v. 31, n. 1, p. 33-55, 2000. http://dx.doi.org/10.1111/j.1540-5915.2000.tb00923.x

KWOK, S.; WU, K. RFID-based intra-supply chain in textile industry. Industrial Management \& Data Systems, v. $109, \quad$ n. 9 , p. 1166-1178, 2009. http://dx.doi. org/10.1108/02635570911002252

LAl, K.; NGAl, E.; CHENG, T. Measures for evaluating supply chain performance in transport logistics. Transportation Research Part E: Logistics and Transportation Review, v. 38, n. 6, p. 439-456, 2002. http://dx.doi.org/10.1016/S13665545(02)00019-4

LAMBERT, D.; POHLEN, T. Supply Chain Metrics. The International Journal of Logistics Management, v. 12, n. 1, p. 1-19, 2001. http://dx.doi.org/10.1108/09574090110806190

LAMMING, R. et al. An initial classification of supply networks. International Journal of Operations \& Production Management, v. 20, n. 6, p. 675-691, 2000. http://dx.doi. org/10.1108/01443570010321667

LORENZONI, G.; LIPPARINI, A. The leverage of interfirm relationships as a distinctive organizational capability. Strategic Management Journal, v. 20, n. 4, p. 317-339, 1999. http://dx.doi. org/10.1002/(SIC1) 1097-0266(199904)20:4\%3C317::AID$\mathrm{SMJ} 28 \% 3 \mathrm{E} 3.0 . \mathrm{CO} ; 2-3$

PIRES, S. Gestão da Cadeia de Suprimentos - Conceitos, Estratégias Práticas e Casos. São Paulo: Atlas, 2004.

RIBEIRO, J.; NEUMANN, C. Planejamento e condução de grupos focados. In: RIBEIRO, J. (Org.) Grupos focados: teoria e aplicações. Porto Alegre: FEENG-UFRGS-PPGEP, 2003.

SAATY, T. Multicriteria Decision Making: the Analytic Hierarchy Process. Pittsburgh: RWS Publications, 1991.

SELLITTO, M.; MENDES, L. Avaliação comparativa do desempenho de três cadeias de suprimentos em manufatura. Produção, v. 16 , n. 3, p. 552-568, 2006. http://dx.doi.org/10.1590/S010365132006000300015

SELLITTO, M.; BORCHARDT, M.; PEREIRA, G. Systemic performance assessment of a kind of complex adaptive system: supply-chains. In: INTERNATIONAL MULTI-CONFERENCE ON COMPLEXITY, INFORMATICS AND CYBERNETICS - IMCIC, 2011, Orlando. Proceedings... Orlando: International Institute of Informatics and Systemics, 2011.

SIMCHI-LEVI, D.; KAMINSKl, P.; SIMCHI-LEVl, E. Cadeia de Suprimentos: Projeto e Gestão. Porto Alegre: Bookman, 2003.

SUPPLY CHAIN COUNCIL - SCC. The. Disponível em: <www.supplychain.com>. Acesso em: ago. 2007.

TAYLOR, D. Logística na cadeia de suprimentos: uma perspectiva gerencial. São Paulo: Pearson, 2006.

THOMAS, D.; GRIFFIN, P. Coordinated supply chain management. European Journal of Operational Research, v. 94, n. 1, p. 1-15, 1996. http://dx.doi.org/10.1016/0377-2217(96)00098-7

YIN, R. Estudo de caso: planejamento e método. Porto Alegre: Bookman, 2001.

WARD, P. et al. Competitive priorities in operations management. Decision Sciences, v. 29, n. 4, p. 1035-1046, 1998. http://dx.doi. org/10.1111/j.1540-5915.1998.tb00886.x

WATTS, C.; KIM, Y.; HAHN, C. linking purchasing to corporate competitive strategy. International Journal of Purchasing and Materials Management, v. 28, n. 4, p. 2-8, 1992.

\title{
Comparative analysis and competitive priorities assessment in three supply chains from the petrochemical industry
}

\begin{abstract}
The purpose of this article is to comparatively analyze and quantitatively assess the competitive priorities in three supply chains from the petrochemical industry: $A, B$, and $C$. The research was carried out in the second half of 2006. The article revises definitions on supply chains with emphasis on network shapes. Then, it presents the three case studies: $A, B$, and $C$. The research identifies seven competitive priorities in the petrochemical industry: quality, lead-time, flexibility, cost, innovation, communication and safety, which were unfolded in dimensions. The priorities were analyzed by managers with the support of the AHP multi-criteria method, reaching a competitiveness structure that supported the assessment based on judgment. Finally, the differences betweenthe priorities and the assessed performances formed a structure of objectives for the chains. Results showed that Ashould prioritize quality, $B$ should prioritize flexibility, while $C$ should prioritize cost reduction.
\end{abstract}

\section{Keywords}

Supply chain. Production strategies. Competitive criteria. Competition in the petrochemical industry. Performance assessment in supply chain. 